\title{
Treatment of Multiple Congenital Peripheral Aneurysms of Upper Extremity - A Case Report
}

\author{
Chandra Sekhar Chevuturu ${ }^{1,}{ }^{*}$, Mahmood Shadab Siddique ${ }^{1}$, Sri Nagesh $^{2}$, Parveen Jindal $^{3}$ \\ ${ }^{1}$ Deparment of Vascular Surgery, Apollo Hospitals, Jubilee Hills, Hyderabad, India \\ ${ }^{2}$ Department of Plastic Surgery,Apollo Hospitals, Jubilee Hills, Hyderabad, India \\ ${ }^{3}$ Renal Care and Dialysis Centre, Inder Road, Dehradun, Uttarakhand, India \\ Email address: \\ chandrasekhar1728@hotmail.com (C. S. Chevuturu)
}

\section{To cite this article:}

Chandra Sekhar Chevuturu, Mahmood Shadab Siddique, Sri Nagesh, Parveen Jindal. Treatment of Multiple Congenital Peripheral Aneurysms of Upper Extremity - A Case Report. Journal of Surgery. Vol. 3, No. 6, 2015, pp. 56-58. doi: 10.11648/j.js.20150306.11

\begin{abstract}
Congenital true aneurisms of peripheral arteries especially on the hand are rare and idiopathic. The present case is focused on the multiple aneurysms of a young boy on both radial and ulnar arteries since birth. The clinical indications include painful ulcers on the right middle finger, discoloration and swelling of dorsum of ulnar side of right hand. In addition, patchy necrosis of fifth finger with increased intensity of pain was observed. Here we successfully treated both aneurysms with partial amputation of the digit and the growth of the skin on both sides of the palm was restored by groin flap.
\end{abstract}

Keywords: Aneurysms, Radial Artery, Ulnar Artery, Groin Flap

\section{Introduction}

Among different Aneurysms, two main types are prevalent namely aortic which include both thoracic \& abdomen and cerebral. In addition to them, pseudo or false aneurysms are also reported frequently. Coming to the aneurysms of distal region of upper extremities, the major causes are trauma and infections[1-2]. But the present case represents a true aneurysm of both radial and ulnar arteries which is congenital and remains as extremely rare and idiopathic in nature. According to the previously suggested clinicopathologicalclassification [3], the present case falls into the category of Class VIII.

We have taken this case to prevent the complete amputation and removed the aneurysms of both arteries and the blood supply was restored to the hand by implanting a synthetic graft as brachio-radial artery bypass. Finally gangrenous skin on the lesions was removed and a groin flap was done for the growth of normal skin on the digit. A formal consent has been obtained from the patient for this report.

\section{Case Report}

A 23 year old male patient presented with complaints of multiple swellings on right forearm and palm since childhood. Since birth, the patient had multiple swellings in his right upper limb which gradually increased in size over the years. He had painful ulcers on the right middle finger, discoloration and swelling of dorsum of ulnar side of right hand with restricted movements of fingers since a year. Recently patchy necrosis of fifth finger was observed and intensity of pain and tenderness in the involved areas in the right upper limb has increased (Fig 1A).Complete amputation of the upper limb was the suggestion given by the contemporary medical fraternity.

He has no history of any previous surgeries, concurrent congenital anomalies, drug/IV abuse, smoking, alcohol consumption, hospitalizations and trauma. He was up to date with his vaccinations. Routine laboratory tests which involved Hepatitis C \& VDRL screening, Chest X-ray, ECG, $2 \mathrm{D}$ Echo has not revealed any complications and infections leaving his systemic examination unremarkable. Angiographic investigation of right upper limb demonstrated a total of four separate saccular true aneurysms, two on radial and two on ulnar arteries near the wrist. There is an extensive collateral supply of hypothenar region of palm supplied by feeders from ulnar artery and also in forearm region.

Under general anasthesia, ulnar artery aneurysms were excised. Since ulnar artery was the feeding artery for the hand swelling, it was ligated. Radial artery aneurysms were excised and blood supply to the hand, was restored by implanting a brachio-radial artery bypass with synthetic graft of $6 \times 40 \mathrm{~cm}$ (Fig 1B and C). Partial amputation of the 
necrosed $5^{\text {th }}$ digit was done and debridement of gangrenous skin over the palmar and dorsal aspect of ulnar side of the hand was completed. Groin flap was placed over the ulnar side of the hand for one month. After a month skin flap was covered and sutured over the ulnar side of the hand. Growth of skin has been observed over the dorsum and on the palmar surface of the hand.
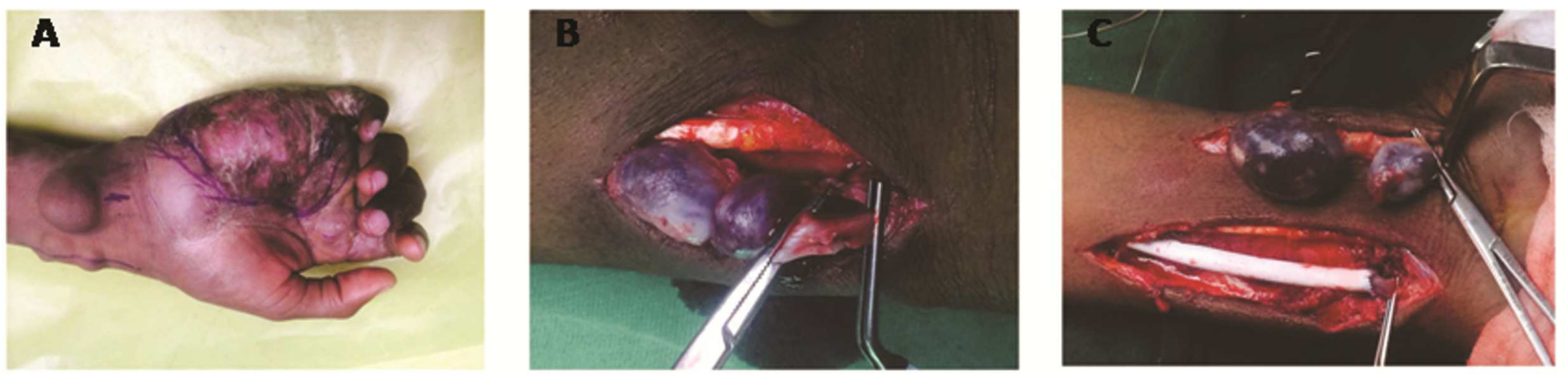

Figure 1. A. Multiple aneurysms along with the swelling of dorsum of the ulnar side of the right hand; $B$. Radial artery clamping before aneurysm excision; $C$. Brachio-Radial artery bypass with synthetic graft after clamping of distal radial artery.
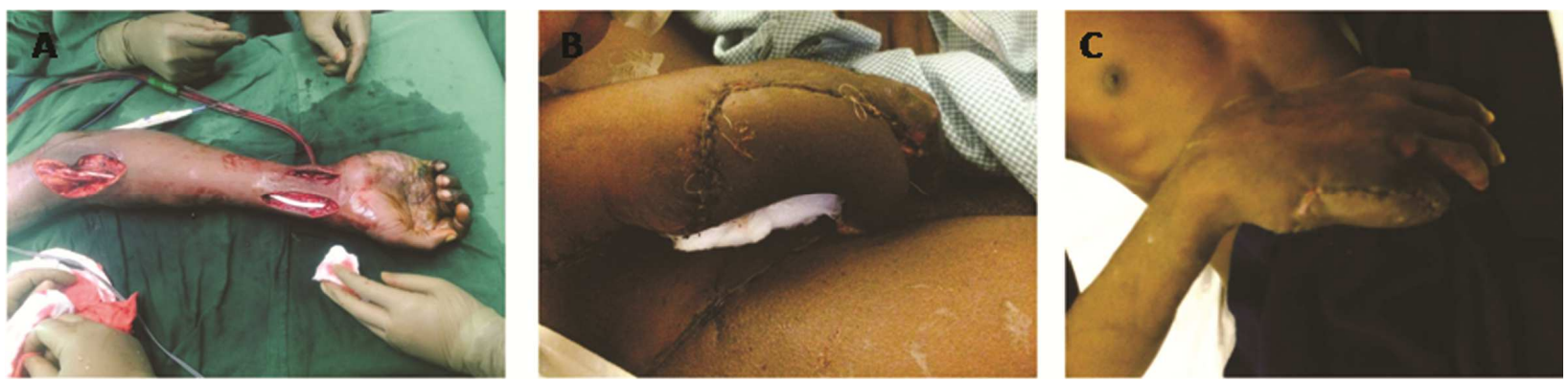

Figure 2. A.Post aneurysms excision and Brachio-radial artery bypass; B.Groin flap covering over the hand; C. Skin growth over dorsum of hand after one month.

\section{Discussion}

Despite of its rarity, occurrence of aneurysm on the hand has been reported previously by many groups and the major causes include trauma, arteriosclerosis, metabolic disorders, diseases etc [4-6]. These include the aneurysms of ulnar, radial and digital arteries which occur individually due to various reasons mentioned above[7-10]. True Congenital appearances of these separate aneurysms are reported very rarely. But present case refers to the most uncommon combination of both true radial and ulnar aneurysms which is congenital in nature.

Even though there were many invasive and noninvasive treatment modalities described previously for different aneurysms, the diagnostic and treatment algorithms for this class of idiopathic aneurysms in arteries are not well established because of their rarity. Since there is a high chance of development of thromboembolic complications with subsequent finger and hand ischemia along with the necrosis in the palm, surgical intervention is inevitable in this case. Surgical options depend on the area and number of aneurysms to be resected and their reconstruction which include arterial ligation, resection of the thrombosed arterial segment, end-to-end anastomosis, and resection and vascular reconstruction with graft [11]. The presence of adequate perfusion in the hand when the aneurysm is excluded also influences the treatment option. Simple resection is sufficient if the hand is adequately perfused and artery is intact whereas artery reconstruction is mandatory if the hand perfusion is inadequate.

The cause of multiple aneurysms in the present case is congenital. The patient appeared normal physically with normal values of the inflammatory markers and the histologic picture of the aneurysm ruled out the possibility of any connective tissue or inflammatory disorders. Due to increase in the necrosis in the palm and the compromised distal run-off, we have chosen an open surgical approach for durable result to avoid the risk for complete amputation of the upper limb. Since the large size of the aneurysm may lead to mechanical compression of adjacent structures, the surgical exploration with removal of hematoma and arterial repair was the most appropriate method. In younger patients, inability in obtaining cultures or biopsies for diagnosis of infection or congenital abnormality and the thrombosis of stent grafts limits of employment of endovascular techniques $[12,13]$.

The treatment is multidisciplinary in nature as it required the collaboration between a vascular and a plastic surgeon. Here we successfully resected all the aneurysms and the ligation of the ulnar artery was done since it is the feeding artery whereas the blood supply in the radial artery could be restored by creating brachio-radial artery bypass using synthetic graft (Fig 2A).The degeneration of almost all allografts used throughout the history of vascular surgery has ultimately prompted us to employ a synthetic graft for the revascularization. Partial amputation of necrosed $5^{\text {th }}$ digit was done followed by the debridement of gangrenous skin. Groin flap was done to 
restore the skin on the ulnar side of the hand for a month which resulted in the efficient skin growth on both the palmar and dorsal surface of the hand (Fig $2 \mathrm{~B}$ and $\mathrm{C}$ ).

\section{Acknowledgements}

Authors acknowledge the participant without whom this study would not have been accomplished.

\section{Funding support}

This study was supported by Apollo Hospitals, India.

\section{References}

[1] Clark ET, Mass DP, Bassiouny HS, Zarins CK, Gewertz BL. True aneurysmal disease in the hand and upper extremity. Ann Vasc Surg 1991; 5: 276-81.

[2] Ho PK, Weiland AJ, Mc Clinton MA, Wilgis EF. Aneurysms of the upper extremity. J Hand Surg 1987; 12: 39-46.

[3] Sarkar R, Coran AG, Cilley RE, Lindenauer SM, Stanley JC. Arterial aneurysms in children: clinicopathologic classification. J Vasc Surg. 1991 Jan; 13 (1): 47-56; discussion $56-7$.
[4] Smith JW. True aneurysms of traumatic origin in the palm. Am J Surg 1962; 104: 7-13.

[5] Ho PK, Weiland AJ, Mc Clinton MA, Wilgis EFS. Aneurysms of the upper extremity. J Hand Surg [Am] 1987; 12: 39-46.

[6] Natali J, Maraval M, Kieffer E. Arterial aneurysms in the upper limbs. J Cardiovasc Surg (Torino) 1973; Suppl: 291-6.

[7] Williams JL. Multiple aneurysms in a child. Proc R Soc Med 1975; 68: 523-5.

[8] Turner WH, Turnbull AR. True radial artery aneurysm.Br J Surg. 1988 Mar; 75(3): 233.

[9] Walton NP, Choudhary F. Idiopathic radial artery aneurysm in the anatomical snuff box. ActaOrthop Belg. 2002 Jun; 68 (3): $292-4$.

[10] Offer GJ, Sully L. Congenital aneurysm of the ulnar artery in the palm. J Hand Surg 1999; 24: 735-7.

[11] Rothkopf DM, Bryan DJ, Cuadros CL, May JW Jr. Surgical management of ulnar artery aneurysms. J Hand Surg 1990; 15: 891-7.

[12] Morrissey NJ. Endovascular treatment of peripheral arterial aneurysms. Mt Sinai J Med. 2004; 71 (1): 1-3.

[13] Buda SJ, Johanning JM. Brachial, radial, and ulnar arteries in the endovascularera: choice of intervention. SeminVasc Surg. 2005; 18 (4): 191-5. 